\title{
Value of Sinogram-Affirmed Iterative Reconstruction for Evaluating Coronary Calcified Plaques
}

\author{
Xianchun Zeng, ${ }^{1,2}$ Shaolei Kang, ${ }^{2}$ Rongpin Wang, ${ }^{1}$ Dan Han,,${ }^{2,}$ Yong Wang, ${ }^{2}$ and Shiping Yang ${ }^{2}$ \\ ${ }^{1}$ Department of Radiology, Guizhou Provincial People's Hospital, Guiyang, China \\ ${ }^{2}$ Department of CT, The First Affiliated Hospital of Kunming Medical University, Kunming, China \\ "Corresponding author: Dan Han, Department of CT, The First Affiliated Hospital of Kunming Medical University, Kunming, P. O. Box: 650032, China. Tel: +86-87165324888, Fax: \\ +86-87165324888, E-mail: danhancn@126.com
}

Received 2014 March 03; Revised 2014 March 08; Accepted 2014 June 22.

\begin{abstract}
Background: Coronary computed tomography angiography (cCTA) technology as a kind of non-traumatic examination has been widely used in clinical practice. There are major issues that need to be considered. One is how to obtain high quality images and at the same time effectively reduce the radiation dose. The second is coronary artery calcified plaque artifacts that seriously affect the depiction of plaque morphology and luminal stenosis. In case of dose reduction, these artifacts are more outstanding.

Objectives: This study determined the value of sinogram-affirmed iterative reconstruction (SAFIRE) technology to assess coronarycalcified plaques. This value was compared with filtered back-projection (FBP) reconstruction.

Patients and Methods: Sixty-three cases with calcified plaques diagnosed via coronary CT examination were selected. The mean CT-number, noise, signal-to-noise ratio (SNR), contrast-to-noise ratio (CNR), number of calcium plaques, edges, lumen situation, and the subjective image quality ratings of the cases using FBP and SAFIRE1-SAFIRE5 (six groups) were analyzed and compared.

Results: The subjective ratings of image quality using SAFIRE1-SAFIRE5 reconstructions were significantly higher than those using FBP, with SAFIRE3 achieving the highest rating. Compared with FBP reconstruction, the differences in noise, SNR, and CNR using SAFIRE1-SAFIRE5 were statistically significant $(\mathrm{P}<0.05)$, with SAFIRE5 reconstruction achieving the highest SNR and CNR, and FBP reconstruction achieving the lowest. The revealed numbers of calcium plaques in the SAFIRE1-SAFIRE5 reconstruction groups were higher than that in the FBP reconstruction without significant differences in the number of calcium plaques among the SAFIRE1-SAFIRE5 groups $(\mathrm{P}>0.05)$.

Conclusion: SAFIRE reconstruction provided better coronary image quality and displayed the number, morphology, and surrounding lumen of calcium plaques more accurately than traditional FBP reconstruction, with SAFIRE3 achieving the best results.
\end{abstract}

Keywords: SAFIRE Reconstruction Technique, cCTA, Calcium Plaque, Noise

\section{Background}

Coronary computed tomography angiography (cCTA), a non-invasive examination technique, is widely used in clinical practice $(1,2)$. However, obtaining high-quality images while effectively reducing the radiation dose is a pressing problem in this technology. Furthermore, the constructed defects of calcified coronary plaques seriously affect plaque morphology and luminal stenosis, and the constructed defects are more prominent at lower radiation doses.

\section{Objectives}

This paper compares the calcium plaques in the coronary artery using sinogram-affirmed iterative reconstruction (SAFIRE), which is a new dual-source CT iterative reconstruction technology and traditional filtered backprojection (FBP) reconstruction technology.

\section{Patients and Methods}

\subsection{Subjects}

A total of 63 patients with coronary calcium plaques who were admitted in the First Affiliated Hospital of Kunming Medical University from August 2012 to September 2012 were selected. These patients were suspected of coronary atherosclerotic heart disease via cCTA examination in the clinic. The patients comprised 39 males and 24 females aged 38 years to 79 years old, with an average age of 65.30 \pm 14.71 years. The inclusion criteria were as follows: body mass index $<25 \mathrm{~kg} / \mathrm{m}^{2}$, total coronary artery calcium score $>0$, and no serious cardiac arrhythmias and significantly increased heart size. This study was conducted in accordance with the declaration of Helsinki after the approval of the Ethics Committee of the 1st Affiliated Hospital of Kunming Medical University. Written informed consent was obtained from all participants and examinations were successfully completed. 


\subsection{Scanning Plan}

The scans were performed using a Siemens Somatom Definition Flash CT (SOMATOM Definition Flash; Siemens Healthcare, Forchheim, Germany). The scan range was from $1 \mathrm{~cm}$ below the tracheal carina to the facies diaphragmatica cordis. The scan parameters were as follows: tube voltage of $100 \mathrm{kV}$, real-time dynamic exposure dose adjustment (CARE Dose 4D) was on, CARE kV Semi (reference voltage and current of 120 and $400 \mathrm{mAs}$, respectively), ECG pulse of $30 \%$ to $75 \%$ and collimator of $128 \times 0.6 \mathrm{~mm}$. Iopromide was injected ( $370 \mathrm{mg} \mathrm{I} / \mathrm{mL}$ ) as contrast agent at a flow rate of $4 \mathrm{~mL} / \mathrm{s}$ to $5 \mathrm{~mL} / \mathrm{s}$ with a total of $50 \mathrm{~mL}$ to $70 \mathrm{~mL}$ and then injected with $30 \mathrm{~mL}$ to $40 \mathrm{~mL}$ of saline for rinsing. Contrast agent bolus tracking software (Bolus Tracking) was used to monitor the plane and select the aortic root. The threshold was set to trigger at $100 \mathrm{HU}$ with a 5 second delay before starting the scan.

\subsection{Post-Processing Program}

The best phase for the coronary scan (phase with minimal motion-constructed defects) was selected and reconstructed using FBP and SAFIRE. The convolution functions were B46f and I46f. The reconstruction thickness was 0.6 $\mathrm{mm}$ with spacing of $0.4 \mathrm{~mm}$. The six sets of data included groups 1-5 in the SAFIRE reconstruction according to rebuild strength. These data were obtained and transmitted into Siemens Syngo Multi-Modality Workplace workstations and then analyzed and measured using Circulation and 3D software.

\subsection{Image Analysis}

Image quality was evaluated subjectively and objectively. The subjective evaluation was performed on 15 modified coronary segments according to the standards of the American Heart Association (3). Two highly qualified physicians blinded to the scan parameters and patient information carried out the double-blind evaluation. Consensus was reached by the physicians through comprehensive analysis in case of any disagreement. Overall image quality scores were divided into four grades as follows (4): 4 points, each coronary segment is displayed clearly, no motion constructed defects and excellent image quality; 3 points, some segments are slightly blurred, mild constructed defects and good image quality; 2 points, general image quality, still continuous coronary structure and moderate constructed defects; and 1 point, poor coronary display, blurred segment that could not be assessed or vascular amputation. Images with scores of at least 2 points were considered diagnostic, i.e.fulfills the diagnostic requirements.
Evaluation of coronary calcium plaques included the number, edge, and adjacent lumen situation. Calcium plaque numbers were compared in the same level and position regardless of size, with all plaques accounted as one unit. The edges and the adjacent lumen situation of the calcium plaques were evaluated based on the scores in the same level, position, and image quality. The scoring scale was as follows: 4 points, no evident constructed defects around the calcium plaques and clear display adjacent to the lumen; 3 points, mild constructed defects around the calcium plaques and clear display adjacent to the lumen; 2 points, moderate constructed defects around the calcium plaques and basically clear lumens; and 1 point, heavy calcium constructed defects around the calcium plaques and unclear lumen.

The objective evaluation indicators included mean CTnumber (Unit is HU), noise, signal-to-noise ratio (SNR) and contrast-to-noise ratio (CNR). The aortic CT was measured from the aortic root (opening plane of the left main coronary artery), and the region of interest (ROI) was $2 \mathrm{~cm}^{2}$. Image noise was measured based on the Standard Deviation (SD) of measured CT, and the average was obtained from the three processing instances of the same image. Coronary average CT, noise, SNR, and CNR (5) were measured based on the following: the average CT values of the coronary ROI in the left main coronary artery, the proximal anterior descending, the anterior descending branch of the distal segment (beyond the second pair of diagonal branch openings), the proximal segment of the first pair diagonal branch, the proximal circumflex artery, the distal circumflex artery (beyond the second pair of obtuse marginal branch openings), the proximal segment of the first obtuse marginal branch, the proximal segment of the right coronary artery (RCA) and the distal segment of the RCA (before posterior descending branch openings), as well as the average CT value of the fat tissue around the left coronary sinus opening. The formulae (6) are as follows: SNR = $\mathrm{CT}$ average $/ \mathrm{SD}$ and $\mathrm{CNR}=(\mathrm{CT}-\mathrm{CT}$ average fat $) / \mathrm{CT}$ fat $\mathrm{SD}$.

\subsection{Radiation Dose}

The volume CT dose index (CTDIvo1), dose-length product $(\mathrm{DLP})$ and effective dose $[(\mathrm{ED})=\mathrm{DLP} \times \mathrm{K}]($ in $\mathrm{mSv})$ were included. $\mathrm{K}$ is the conversion factor, and the European Quality Standard Guide proposed CT chest average of 0.014 $\mathrm{mSv} /(\mathrm{mGy} \mathrm{cm})$ was used (7).

\subsection{Statistical Analysis}

SPSS Ver 17.0 package (Statistical Product and Service Solutions, IBM, USA) was used for statistical analysis. The CT values, SNR, CNR and the number of calcium plaques in the images of the six groups were displayed and compared 
using ANOVA. The rank of the classification data was compared using a multi-group ordinal analysis. The overall image quality score and the ratings of calcium spots and surrounding lumen were compared using the $\chi^{2}$ test. $\mathrm{P}<0.05$ was considered statistically significant.

\section{Results}

\subsection{Image Quality Scores}

The subjective image quality scores of the six groups scored at least two. The $\chi^{2}$ test was performed, and $\mathrm{P}<$ 0.05 indicated that the difference was statistically significant. Further pairwise comparisons were conducted, but no significant differences were observed between FBP and SAFIRE1-5. By contrast, the differences were significant between FBP and SAFIRE2, and SAFIRE3 and SAFIRE4. No significant differences were found between SAFIRE2, SAFIRE3 and SAFIRE4 scores. SAFIRE3 exhibited the highest subjective score (3.23 \pm 0.52$)$ (Table 1 ).

Table 1. Comparisons of Subjective Ratings for Image Quality in Different Reconstruction Modes (Points)

\begin{tabular}{|c|c|c|}
\hline Variables & Scoring & PValue \\
\hline Reconstruction Modes & & 0.036 \\
\hline FBP & $2.72 \pm 0.57$ & \\
\hline SAFIRE-1 & $2.71 \pm 0.58$ & \\
\hline SAFIRE-2 & $3.11 \pm 0.49$ & \\
\hline SAFIRE-3 & $3.23 \pm 0.52$ & \\
\hline SAFIRE-4 & $3.09 \pm 0.56$ & \\
\hline SAFIRE-5 & $2.69 \pm 0.53$ & \\
\hline
\end{tabular}

Abbreviations: FBP, filtered back projection; SAFIRE, sinogram-affirmed iterative reconstruction.

\subsection{Comparisons of Calcium Plaque Display}

The F test was used to compare the number of calcium plaques. The F value was 16.20 , and the result was statistically significant at $\mathrm{P}<0.05$. The results of further pairwise comparisons showed the differences among the groups using FBP and SAFIRE reconstructions. The calcium plaques in SAFIRE1-SAFIRE5 (I46f) reconstructions were higher than that in the FBP (B26f) reconstruction, whereas the SAFIRE1-SAFIRE5 reconstructions (I46f) exhibited no significant differences. The $\mathrm{F}$ test was performed to compare calcium plaque edges and adjacent lumen ratings. The $\mathrm{F}$ values were 16.78 and 17.22 at $\mathrm{P}<$ 0.05 , which were statistically significant. Further pairwise comparisons showed that the differences between FBP scores and SAFIRE1-SAFIRE5 scores were statistically significant, whereas no significant differences were observed between SAFIRE1 and SAFIRE5 scores, as well as between SAFIRE2, SAFIRE3 and SAFIRE4 scores. No significant differences were observed between SAFIRE2, SAFIRE3 and SAFIRE4 scores. SAFIRE3 exhibited the highest subjective scores. The score for calcium plaque edge was $3.33 \pm 0.22$, and the displayed rating of calcium plaque adjacent lumen was $3.35 \pm 0.26$ (Table 2). This result indicates that SAFIRE3 reconstruction displayed the clearest edges of calcium plaque and adjacent lumen (Figure 1A-1E).

Table 2. Displays on Calcium Plaques of Different Reconstructed Images

\begin{tabular}{|lccc}
\hline $\begin{array}{l}\text { Reconstruction } \\
\text { Modes }\end{array}$ & $\begin{array}{c}\text { Number of } \\
\text { Calcium } \\
\text { Plaques }(\mathbf{n})\end{array}$ & $\begin{array}{c}\text { Edges of } \\
\text { Calcium } \\
\text { Plaques } \\
\text { (Scores) }\end{array}$ & $\begin{array}{c}\text { Display of the } \\
\text { Adjacent } \\
\text { Lumens to } \\
\text { Calcium } \\
\text { Plaques } \\
\text { (Scores) }\end{array}$ \\
\hline FBP & 393 & $2.93 \pm 0.32$ & $2.89 \pm 0.30$ \\
\hline SAFIRE-1 & 397 & $3.01 \pm 0.25$ & $3.05 \pm 0.22$ \\
\hline SAFIRE-2 & 397 & $3.12 \pm 0.21$ & $3.14 \pm 0.25$ \\
\hline SAFIRE-3 & 397 & $3.33 \pm 0.22$ & $3.35 \pm 0.26$ \\
\hline SAFIRE-4 & 397 & $3.18 \pm 0.25$ & $3.17 \pm 0.24$ \\
\hline SAFIRE-5 & 397 & $3.07 \pm 0.27$ & $3.03 \pm 0.25$ \\
\hline F value & 16.20 & 16.78 & 17.22 \\
\hline P value & $<0.05$ & $<0.05$ & $<0.05$ \\
\hline
\end{tabular}

Abbreviations: FBP, filtered back projection; SAFIRE, sinogram-affirmed iterative reconstruction.

\subsection{Comparisons of Average CTValue, SNR and CNR}

The mean CT, SNR and CNR of the six data sets were compared using the F test. No significant differences were observed in the aortic root, RCA, left anterior descending (LAD), and average CT values of the suncutaneous fat scanning plane ( $\mathrm{P}>0.05)$, and this trend was the same in different parts. The SNR, CNR comparisons of the aortic root, RCA and LAD showed statistically significant differences $(\mathrm{P}<0.05)$. Further pairwise comparisons between the two groups were statistically significant. The SAFIRE5 reconstruction exhibited the highest SNR and CNR, whereas those of the FBP reconstruction were the lowest (Table 3).

\subsection{Radiation Dose}

In this group, the average CTDIvol, DLP and ED were $46.84 \pm 5.00$ (mGy), $244.12 \pm 13.53(\mathrm{mGy} \times \mathrm{cm})$, and $3.41 \pm$ 0.19 ( $\mathrm{mSv}$ ), respectively. 

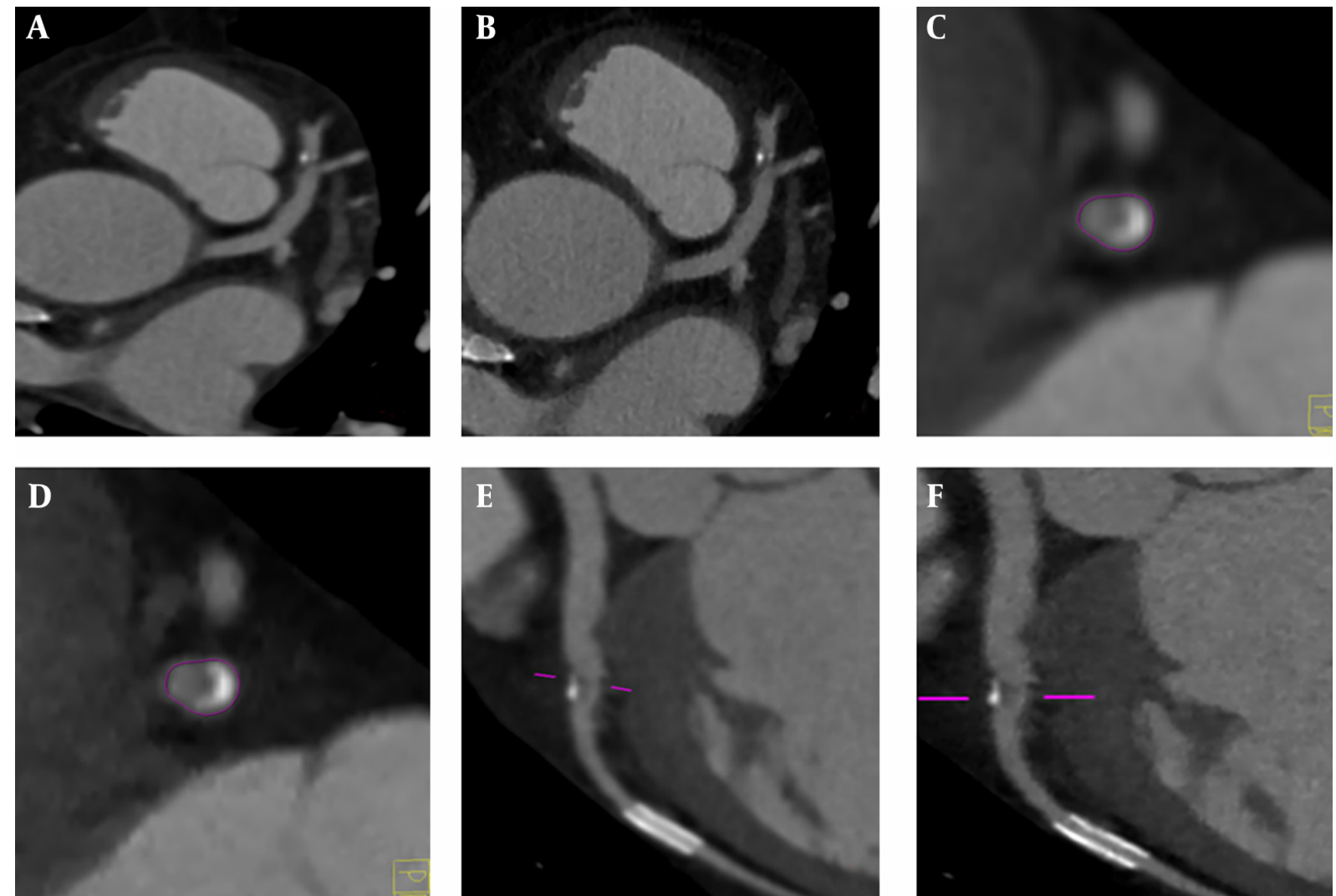

Figure 1. 1. A-D, These images are in the same patient with a heart rate of 73 beats/minutes, BMI of $23.6(\mathrm{~kg} / \mathrm{m} 2)$, and scan parameters of $120 \mathrm{kV}$ and $352 \mathrm{mAs}$. The cross-sectional reconstruction of data in SAFIRE3 and FBP at the same level displayed that the images of calcium plaques in SAFIRE3 were clearer, especially the small calcium spots adjacent to calcium plaques. E-F, are the same patient, with a heart rate of 75 beats $/$ minute, BMI of $24.1(\mathrm{~kg} / \mathrm{m} 2)$, and scan parameters of $120 \mathrm{kV}$ and $352 \mathrm{mAs}$. The curved surface reconstruction of data in SAFIRE3 and FBP at the same level displayed that the images of calcium plaques and the adjacent lumens in SAFIRE3 were clearer than that in FBP reconstruction. (BMI, body mass index; FBP, filtered back-projection; SAFIRE, sinogram-affirmed iterative reconstruction).

Table 3. Comparisons of Average CT Value in Different Reconstructed Images

\begin{tabular}{|c|c|c|c|c|c|c|c|}
\hline \multirow{2}{*}{ Reconstruction Modes } & \multicolumn{3}{|c|}{ Aorta } & \multicolumn{3}{|c|}{ RCA } & \multirow{2}{*}{ CNR (RCA) } \\
\hline & CT Value (HU) & SD & SNR & CT Value (HU) & SD & SNR & \\
\hline FBP & $453.25 \pm 4.33$ & $45.33 \pm 0.43$ & $10.00 \pm 2.10$ & $459.65 \pm 6.22$ & $47.14 \pm 0.64$ & $9.75 \pm 2.01$ & $13.68 \pm 1.68$ \\
\hline SAFIRE-1 & $460.50 \pm 3.21$ & $28.09 \pm 0.19$ & $16.39 \pm 2.11$ & $464.37 \pm 7.18$ & $30.02 \pm 0.46$ & $15.47 \pm 2.14$ & $17.48 \pm 2.35$ \\
\hline SAFIRE-2 & $462.55 \pm 3.51$ & $25.43 \pm 0.19$ & $18.19 \pm 2.14$ & $467.15 \pm 6.54$ & $26.57 \pm 0.37$ & $17.58 \pm 2.10$ & $19.35 \pm 2.48$ \\
\hline SAFIRE-3 & $461.25 \pm 3.37$ & $20.87 \pm 0.12$ & $22.10 \pm 2.16$ & $462.58 \pm 7.45$ & $22.18 \pm 0.36$ & $20.86 \pm 2.07$ & $21.26 \pm 2.65$ \\
\hline SAFIRE-4 & $462.17 \pm 4.48$ & $16.36 \pm 0.17$ & $28.25 \pm 0.27$ & $460.44 \pm 6.61$ & $17.77 \pm 0.26$ & $25.91 \pm 0.03$ & $27.29 \pm 3.49$ \\
\hline SAFIRE-5 & $457.81 \pm 4.44$ & $13.63 \pm 0.13$ & $33.59 \pm 0.18$ & $461.30 \pm 6.37$ & $15.51 \pm 0.20$ & $31.79 \pm 0.03$ & $29.70 \pm 3.73$ \\
\hline Fvalue & 3.32 & 2.74 & 2.11 & 3.12 & 2.76 & 2.65 & 3.08 \\
\hline Pvalue & 0.40 & 0.21 & 0.15 & 0.36 & 0.23 & 0.18 & 0.34 \\
\hline
\end{tabular}

Abbreviations: FBP, filtered back projection; SAFIRE, sinogram-affirmed iterative reconstruction; SD, standard deviation; SNR, signal-to-noise ratio; CNR, contrast-to-noise ratio; RCA: right coronary artery; HU, Hounsfield Unit.

\section{Discussion}

Analytic reconstruction (AR) and iterative reconstruction (IR) are two basic methods for CT image reconstruc- tion. The AR model is based on continuous signal, which is sensitive to noise and requires complete projection data. 
FBP is the main algorithm for AR. An IR model is based on a discrete signal, which can reconstruct better quality images compared with FBP despite low SNR and incomplete projection data (5). However, the large storage space and the long time required by IR limit its application $(6,7)$. Although IR reconstruction was used in the world's first medical CT, this technology was immediately replaced by FBP, which became the 'gold standard' for image reconstruction (8). IR is widely applied because of the rapid improvement in computer performance. Studies confirm that IR reconstruction has better image quality for chest and abdominal scans, less image noise and potentially requires lower radiation doses than conventional FBP reconstruction (9-11). Studies have reported (12-14) that beam hardening-constructed defects can be reduced using IR, which is advantageous in cardiovascular imaging, and can be used to evaluate the condition of hard plaques and coronary arteries. SAFIRE reconstruction established based on IR technology from the Siemens Company consisted of two processes. The first process corrects the raw data by removing or reducing constructed defects from beam hardening. This process reduces image noise through several cycles, but causes no evident change in image sharpness. The second process reduces the statistical optimization of the image noise, which involves iterative correction on an image space. SAFIRE-reconstructed images are constantly compared with the original image, and the reconstruction process continuously cycles according to the inspection type until the best quality images are reconstructed (15).

Numerous studies have been conducted on CCTA (14, 16), but mostly limited on investigating scanning speed, scanning dose, contrast agent dose and other aspects. Few reports have focused on removing or reducing the constructed defects of calcium plaques. These defects are mainly due to the effects of dose reduction and more prominent calcium plaque edges on the images of the calcium plaques and their surroundings (17-19). In the current study, 393 and 397 calcium plaques were found using FBP and SAFIRE reconstructions, respectively. The difference was significant $(\mathrm{P}<0.05)$, but the number of plaques in the SAFIRE1-SAFIRE5 groups did not significantly differ $(P>0.05)$. These findings indicate that SAFIRE reconstruction displayed more sensitively calcium plaques, particularly small calcium plaques, than the traditional FBP reconstruction, thereby providing more direct signs for detecting calcium plaques and mixed calcium plaques. The ratings of calcium plaque edges and the adjacent luminal stenosis showed that all SAFIRE1-SAFIRE5 scores were significantly higher than the FBP scores. The higher scores suggested that SAFIRE reconstruction reduced the constructed defects caused by calcium plaques compared with the conventional FBP reconstruction. This phenomenon greatly increased the clarity of calcium plaque edges and improved the accuracy of assessing the stenosis caused by calcium plaques or mixed calcium plaques. This advantage is important for disease assessment and treatment guidance. SAFIRE3 achieved the highest subjective score with the best effect, which is consistent with the rebuild strength recommended by the manufacturer, and most reports compared SAFIRE3 with FBP reconstruction (20). The changes in CT did not significantly differ between these groups using the same organization, but the overall image score, SD, SNR, and CNR of the SAFIRE reconstruction of different parts differed significantly. Image noise was reduced by $46.9 \%$ in the SAFIRE1 reconstruction, $54.3 \%$ in the SAFIRE2 reconstruction, $64.3 \%$ in the SAFIRE3 reconstruction, 69.3\% in the SAFIRE4 reconstruction, and 76.1\% in the SAFIRE5 reconstruction. SAFIRE5 achieved the greatest noise reduction and improvement in image quality at an SNR of $75.7 \%$ and a CNR of $58.3 \%$. However, it had lower subjective evaluation scores than the FBP reconstruction. This discrepancy is possibly attributed to the noise characteristics and features of constructed defects in SAFIRE, which differed from that of FBP reconstruction because SAFIRE uses a statistically optimal criterion, but radiologists are used to viewing FBP images.

In summary, SAFIRE reconstruction displayed the number and morphology of calcified plaques and the surrounding lumen more accurately than the traditional FBP reconstruction, with SAFIRE3 achieving the best results. In addition, SAFIRE reconstruction effectively reduced noise and improved image quality, thereby potentially lowering the required dose. However, this study has some limitations. First, it lacked controls for the golden standard in terms of the number of plaques and the degree of stenosis. Second, the selection of subjective ratings may differ because doctors are used to viewing FBP reconstructed images. These limitations will be addressed in subsequent studies.

\section{Footnotes}

Authors' Contribution: Dan Han designed the study. Shaolei Kang and Rongpin Wang performed the study. Yong Wang and Shiping Yang analyzed the data. Xianchun Zeng wrote the paper. All authors read and approved the final manuscript.

Funding/Support: This work was supported by the Social Development Project of Guizhou Province (QKH SY [2011] No. 3057) and Scientific Research Fund Project of the Department of Education, Yunnan Province (2010Y177). 


\section{References}

1. La Grutta L, Runza G, Galia M, Maffei E, Lo Re G, Grassedonio $\mathrm{E}$, et al. Atherosclerotic pattern of coronary myocardial bridging assessed with CT coronary angiography. Int J Cardiovasc Imaging. 2012;28(2):405-14. doi: 10.1007/s10554-011-9817-2. [PubMed: 21347597].

2. Dharampal AS, Rossi A, de Feyter PJ. Computed tomographycoronary angiography in the detection of coronary artery disease. J Cardiovasc Med (Hagerstown). 2011;12(8):554-61. doi: 10.2459/JCM.ob013e32834905dc. [PubMed: 21709578].

3. Alkadhi H, Scheffel H, Desbiolles L, Gaemperli O, Stolzmann P, Plass A, et al. Dual-source computed tomography coronary angiography: influence of obesity, calcium load, and heart rate on diagnostic accuracy. Eur Heart J. 2008;29(6):766-76. doi:10.1093/eurheartj/ehn044. [PubMed: 18292596].

4. Herzog BA, Husmann L, Burkhard N, Valenta I, Gaemperli O, Tatsugami $\mathrm{F}$, et al. Low-dose CT coronary angiography using prospective ECG-triggering: impact of mean heart rate and heart rate variability on image quality. Acad Radiol. 2009;16(1):15-21. doi: 10.1016/j.acra.2008.06.010. [PubMed: 19064207].

5. Paul JF. Individually adapted coronary 64-slice CT angiography based on precontrast attenuation values, using different $\mathrm{kVp}$ and tube current settings: evaluation of image quality. Int J Cardiovasc Imaging. 2011;27 Suppl 1:53-9. doi: 10.1007/s10554-011-9960-9. [PubMed: 22005891].

6. Ripsweden J, Brismar TB, Holm J, Melinder A, Mir-Akbari H, Nilsson T, et al. Impact on image quality and radiation exposure in coronary CT angiography: $100 \mathrm{kVp}$ versus $120 \mathrm{kVp}$. Acta Radiol. 2010;51(8):903-9. doi: 10.3109/02841851.2010.504740. [PubMed: 20735275].

7. Hausleiter J, Martinoff S, Hadamitzky M, Martuscelli E, Pschierer I, Feuchtner GM, et al. Image quality and radiation exposure with a low tube voltage protocol for coronary CT angiography results of the PROTECTION II Trial. JACC Cardiovasc Imaging. 2010;3(11):1113-23. doi: 10.1016/j.jcmg.2010.08.016. [PubMed: 21070998].

8. Slovis TL. CT and computed radiography: the pictures are great, but is the radiation dose greater than required?. AJR Am J Roentgenol. 2002;179(1):39-41. doi: 10.2214/ajr.179.1.1790039. [PubMed: 12076901].

9. Gosling O, Loader R, Venables P, Roobottom C, Rowles N, Bellenger $\mathrm{N}$, et al. A comparison of radiation doses between state-of-the-art multislice CT coronary angiography with iterative reconstruction, multislice CT coronary angiography with standard filtered backprojection and invasive diagnostic coronary angiography. Heart. 2010;96(12):922-6. doi: 10.1136/hrt.2010.195909. [PubMed: 20538667].

10. Prakash P, Kalra MK, Ackman JB, Digumarthy SR, Hsieh J, Do S, et al. Diffuse lung disease: $\mathrm{CT}$ of the chest with adaptive statistical iterative re- construction technique. Radiology. 2010;256(1):261-9. doi: 10.1148/radiol.10091487. [PubMed: 20574099].

11. Moscariello A, Takx RA, Schoepf UJ, Renker M, Zwerner PL, O’Brien TX, et al. Coronary CT angiography: image quality, diagnostic accuracy, and potential for radiation dose reduction using a novel iterative image reconstruction technique-comparison with traditional filtered back projection. Eur Radiol. 2011;21(10):2130-8. doi: 10.1007/s00330011-2164-9. [PubMed: 21611758].

12. Han BK, Grant KL, Garberich R, Sedlmair M, Lindberg J, Lesser JR. As sessment of an iterative reconstruction algorithm (SAFIRE) on image quality in pediatric cardiac CT datasets. J Cardiovasc Comput Tomogr 2012;6(3):200-4. doi: 10.1016/j.jcct.2012.04.008. [PubMed: 22682262].

13. Ebersberger U, Tricarico F, Schoepf UJ, Blanke P, Spears JR, Rowe GW, et al. CT evaluation of coronary artery stents with iterative image reconstruction: improvements in image quality and potential for radiation dose reduction. Eur Radiol. 2013;23(1):125-32. doi: 10.1007/s00330-0122580-5. [PubMed: 22777622].

14. Wang R, Schoepf UJ, Wu R, Reddy RP, Zhang C, Yu W, et al. Image quality and radiation dose of low dose coronary CT angiography in obese patients: sinogram affirmed iterative reconstruction versus filtered back projection. Eur J Radiol. 2012;81(11):3141-5. doi: 10.1016/j.ejrad.2012.04.012. [PubMed: 22578834].

15. Karaca M, Kirilmaz A, Oncel G, Oncel D, Yilmaz H, Tamci B, et al. Contrast-enhanced 64-slice computed tomography in detection and evaluation of anomalous coronary arteries. Tohoku J Exp Med. 2007;213(3):249-59. [PubMed: 17984622].

16. Fei X, Du X, Li P, Liao J, Shen Y, Li K. Effect of dose-reduced scan protocols on cardiac coronary image quality with 64-row MDCT: a cardiac phantom study. Eur J Radiol. 2008;67(1):85-91. doi: 10.1016/j.ejrad.2007.07.008. [PubMed:17765422].

17. Achenbach S, Goroll T, Seltmann M, Pflederer T, Anders K, Ropers D, et al. Detection of coronary artery stenoses by low-dose, prospectively ECG-triggered, high-pitch spiral coronary CT angiography.JACC Cardiovasc Imaging. 2011;4(4):328-37. doi: 10.1016/j.jcmg.2011.01.012. [PubMed: 21492807].

18. Gagarina NV, Irwan R, Gordina G, Fominykh E, Sijens PE. Image quality in reduced-dose coronary CT angiography. Acad Radiol. 2011;18(8):984-90. doi: 10.1016/j.acra.2011.03.009. [PubMed: 21652231].

19. Goetti R, Leschka S, Boschung M, Mayer S, Wyss C, Stolzmann P, et al. Radiation doses from phantom measurements at high-pitch dualsource computed tomography coronary angiography. Eur J Radiol 2012;81(4):773-9. doi:10.1016/j.ejrad.2011.01.068. [PubMed: 21310565].

20. Winklehner A, Karlo C, Puippe G, Schmidt B, Flohr T, Goetti R, et al. Raw data-based iterative reconstruction in body CTA: evaluation of radiation dose saving potential. Eur Radiol. 2011;21(12):2521-6. doi: 10.1007/s00330-011-2227-y. [PubMed: 21822785]. 